\title{
Effect of latex conversion on glass transition temperature
}

\author{
Shao-Xiang Li ${ }^{1}$, Ying-Dong Guan ${ }^{1 *}$, Lu-Mei Liü \\ ${ }^{1}$ Department of Micropolymer Materials of Science and Engineering, Qingdao University of Science \& Technology, Qingdao, China; \\ *Corresponding Author: gyd821123@126.com \\ ${ }^{2}$ Department of Materials Science and Engineering, Qingdao University of Science \& Technology, Qingdao, China
}

Received 29 January 2010; revised 1 March 2010; accepted 25 March 2010.

\begin{abstract}
We have synthesized styrene-acrylic latex and investigated the effect of such reaction conditions as the dosage of initiator, surfactant and stirring speed on monomer conversion and glass transition temperature $(\mathrm{Tg})$ of polymer by means of orthogonal experiment, then we get the best reaction conditions. Test results prove that the glass transition temperature of the polymer is directly related to the monomer conversion. The improvement of monomer conversion can make the glass transition temperature close to the theoretical value. In the case of high final conversion, we can predict the glass transition temperature of the polymers of different composition according to the theoretical relation effectively.
\end{abstract}

Keywords: Monomer Conversion; Orthogonal Experiment; Glass Transition Temperature

\section{INTRODUCTION}

Styrene-acrylic latex is made of styrene and acrylate monomers, which has many advantages. For example, it has wide source of raw materials, high function/price ratio, simple synthetic process and the latex has outstanding water resistance, alkali resistance, scrub resistance and also the paint film has good outdoor durable, adhesive attraction. So the styrene-acrylic latex has been widely used in building coating, metal surface coating and so on. Many researchers [1-5] have studied styrene-acrylic latex. Climates are usually diverse across countries, even in one country. Therefore, a single recipe cannot satisfy different needs in the different climate. In order to adapt to different environment, especially the temperature environment, it requires the minimum film-forming temperature can not only has an unchangeable temperature. Scholars in this area had focused mostly on performance optimi- zation but ignored the investigation of minimum filmforming temperature. In fact, there is a big difference between actual minimum film-forming temperature and theoretical minimum film-forming temperature, which brings polymer designers difficulties in predicting glass transition temperature and designing the hardness of the polymer, at the same time, brings users a lot of inconvenience in use. There are many reasons for the difference between actual minimum filmforming temperature and theoretical minimum filmforming temperature, one of the most important is the monomer conversion. Due to the minimum filmforming temperature has a good corresponding relation with the glass-transition temperature [6], so this paper mainly investigates the glass-transition temperature by means of optimizing the latex's polymerization conditions. We obtain latex with high conversion, thus we can solve the above problems in polymerization technology aspect and obtain the latex recipe of different glass transition temperature under the guidance of the theoretical relation.

\section{EXPERIMENTAL}

\subsection{Materials}

Butyl acrylate (BA, 96\%), Styrene (St, 97\%), Methyl Methacrylate (MAA, 96\%) and Diacetone acryl amide (DAAM) were purchased from Qingdao Reagent Company. The anionic surfactant sodium dodecyl sulfate (SDS), nonionic surfactant nonylphenol polyoxyethylene ether (OP-10) and ammonium persulfate (APS) were purchased from Qingdao Chemistry Reagent Company. All materials were used without further purification.

\subsection{Preparation of Styrene-Acrylic Latex}

All emulsifier and deionized water were feeded into four-necked flask and stirred at high speed first, then feed monomer mixtures slowly to obtain the beforehand latex. Take part of beforehand latex for seed latex, when temperature was wormed up to $75 \pm 1{ }^{\circ} \mathrm{C}$, feed 
part of initiator solution. After the blue seed latex formed, the remaining latex was fed gradually, and partially drop initiator, beforehand latex and initiator were added respectively in $3.5 \mathrm{~h}$ and $4 \mathrm{~h}$. Then the temperature was heated to $85 \pm 1{ }^{\circ} \mathrm{C}$ and kept this temperature for $1 \mathrm{~h}$, then cooled, adjusted $\mathrm{PH}=7-8$, Filtered and Collected latex at last.

\subsection{Characterization}

The test of solid content:

$$
s=\frac{m_{1}}{m_{0}} \times 100 \%
$$

where $s$ is the solid content of latex, $m_{1}$ is the weight of the latex after dried at $80^{\circ} \mathrm{C}$ in vacuum drying oven, $m_{0}$ is the weight of the latex.

The test of monomer conversion:

We calculate the conversion by below relation

$$
C(\%)=\frac{W_{1} \times S+W_{2}-W_{3}-W_{4}}{W_{0}} \times 100 \%
$$

where $W_{1}$ is the whole output of latex, $W_{2}$ is the amount of gel, $W_{3}$ is the amount of initiator, $W_{4}$ is the amount of emulsifier, $W_{0}$ is the amount of whole monomers, $S$ is the solid content of latex.

The theoretical value of copolymer's glass transition temperature:

Using the following FOX relation, we can get the composition of copolymers which have an expectable $\mathrm{Tg}$.

$$
\frac{1}{T g}=\frac{W_{1}}{T g_{1}}+\frac{W_{2}}{T g_{2}}+\frac{W_{3}}{T g_{3}}+\ldots \ldots \frac{W_{n}}{T g_{n}}
$$

where $\mathrm{Tg}$ is the glass transition temperature of copolymers, $T g_{1}, T g_{2}, T g_{3}, T g_{n}$ are the glass transition temperature of the respective homopolymers and $W_{1}$, $W_{2}, W_{3}, W_{\mathrm{n}}$ are the weight fraction of the respective groups.

\subsection{Differential Scanning Calorimeter (DSC) Analysis}

Tg was measured by the DSC method in a NEYZSCH 204F1 type differential scanning calorimeter for polymer samples of $\sim 20 \mathrm{mg}$. DSC condition measurement: hold for $1.0 \mathrm{~min}$ at $-100^{\circ} \mathrm{C}$, heat from -80 to $100^{\circ} \mathrm{C}$ at $10^{\circ} \mathrm{C} \mathrm{min}{ }^{-1}$ and with nitrogen protection.

\section{RESULTS AND DISCUSSION}

\subsection{Choice of Variables and Level of the Orthogonal Experiment and its Results}

During the experiment we found that there is a big difference between measured value and theoretical value of $\mathrm{Tg}$ (the theoretical value is calculated by FOX relation).
After analysis, the author believes that the main reason of this phenomenon is due to a lower conversion of polymerization, the system was not polymerized according to the expectable proportion, so we do the experiment to optimize the process parameters of polymerization by orthogonal experimental firstly in order to obtain the latex with high monomer conversion.

Based on a large number of references and many repeated experiments, we consider that the dosage of initiator (A), Emulsifier (B) and stirring speed (C) are the main factors of polymerization, and have designed L9 $\left(3^{3}\right)$ orthogonal table (three variables, three levels Orthogonal design), the results are shown in Table 1.

\subsection{The Analysis of Orthogonal Experiment Results}

The weighted average $(K)$ and range $(\mathrm{R})$ are given in Table 2.

Table 2 shows that the sequence of the effect of various factors on conversion is emulsifier $>$ initiator $>$ stirring speed, the best condition is $\mathrm{A}_{3} \mathrm{~B}_{2} \mathrm{C}_{2}$ : initiator: $0.8 \%$, emulsifier: $4 \%$, stirring speed: $180 \mathrm{rpm}$. But we can see that the difference between $k_{2}(88.033)$ and $k_{3}(89.000)$ is very small, and as we know the conversion increase with the increase of the initiator, but the gel will increase obviously and the polymerization will become unstable at the same time, so we choose $\mathrm{A}_{2} \mathrm{~B}_{2} \mathrm{C}_{2}$ : initiator: $0.6 \%$, emulsifier: $4 \%$, stirring speed: $180 \mathrm{rpm}$ at last.

Table 1. Test results.

\begin{tabular}{ccccc}
\hline $\begin{array}{c}\text { Test } \\
\text { NO }\end{array}$ & $\begin{array}{c}\mathrm{A} \\
\%\end{array}$ & $\begin{array}{c}\mathrm{B} \\
\%\end{array}$ & $\begin{array}{c}\mathrm{C} \\
\mathrm{rpm}\end{array}$ & $\begin{array}{c}\text { Conversion } \\
\%\end{array}$ \\
\hline 1 & $1(0.4)$ & $1(2)$ & $1(140)$ & 82.6 \\
2 & 1 & $2(4)$ & $2(180)$ & 90.0 \\
3 & 1 & $3(6)$ & $3(240)$ & 78.3 \\
4 & $2(0.6)$ & 1 & 2 & 87.6 \\
5 & 2 & 2 & 3 & 92.5 \\
6 & 2 & 3 & 1 & 84.0 \\
7 & $3(0.8)$ & 1 & 3 & 90.0 \\
8 & 3 & 2 & 1 & 91.0 \\
9 & 3 & 3 & 2 & 86.0 \\
\hline
\end{tabular}

Table 2. The analysis of experiment results.

\begin{tabular}{ccccc}
\hline Test Indicators & & $\mathrm{A}$ & $\mathrm{B}$ & $\mathrm{C}$ \\
\hline \multirow{3}{*}{ conversion } & $k_{1}$ & 83.633 & 86.733 & 85.867 \\
& $k_{2}$ & 88.033 & 91.167 & 87.867 \\
& $k_{3}$ & 89.000 & 82.767 & 86.933 \\
& $\mathrm{R}$ & 5.367 & 8.400 & 2.000 \\
\hline
\end{tabular}

Table 3. Properties of the latex under the condition of $\mathrm{A}_{2} \mathrm{~B}_{2} \mathrm{C}_{2}$.

\begin{tabular}{cccc}
\hline solid content (\%) & gel (\%) & water absorption(\%) & Conversion(\%) \\
\hline 48.6 & 3.5 & 7 & 94 \\
\hline
\end{tabular}


As shown in Tables 1 and $\mathbf{2}$, the final conversion can reach $94 \%$ under the condition of $\mathrm{A}_{2} \mathrm{~B}_{2} \mathrm{C}_{2}$ and it is higher than the others, in addition, some other properties are ideal too.

\subsection{DSC Test Analysis}

In this paper, the initial composition of monomer is BA: MMA:St $=33: 19: 18$, the theoretical value of $\mathrm{Tg}$ which is calculated by FOX relation is $8^{\circ} \mathrm{C}$. But when we adopt the flowing factor: initiator: $0.6 \%$, emulsifier: $4 \%$, speed: $180 \mathrm{rpm}$, the final conversion reaches $94 \%$ and the measured value of $\mathrm{Tg}$ achieved by DSC test is $9.8^{\circ} \mathrm{C}$ (Figure 1), the difference between them is small. That is to say at the condition of high conversion, the measured value of $\mathrm{Tg}$ is very close to its theoretical value and so we can design the hardness of copolymers according FOX relation.

\subsection{The Relationship of Monomer Conversion and Glass Transition Temperature}

Figure 2 shows us the relation between $\mathrm{Tg}$ and monomer conversion (BA:MMA:St $=33: 19: 18)$ and we can

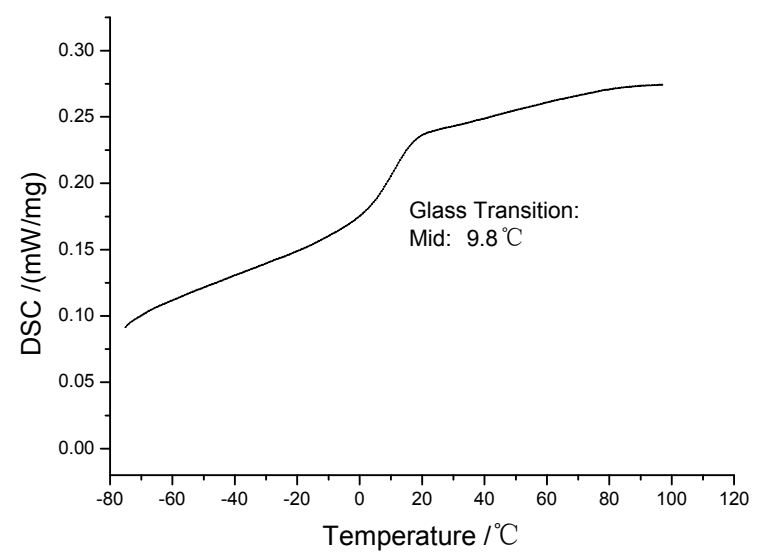

Figure 1. DSC curve of Styrene-acrylic latex (BA:MMA:St = 33:19:18).

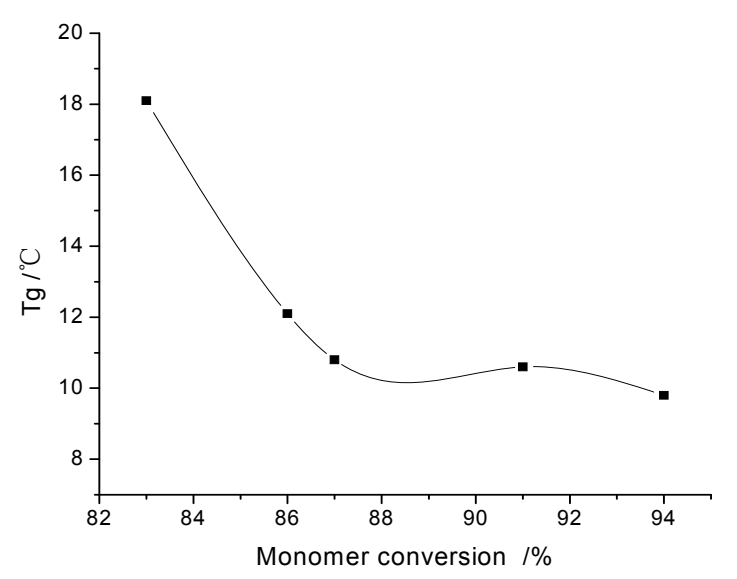

Figure 2. The relation between $\mathrm{Tg}$ and monomer conversion. see from it that with the increase of the monomer conversion, the glass transition temperature of the latex decrease gradually, when the final conversion is over $90 \%$, $\mathrm{Tg}$ reaches a plateau and the value is about $10.0^{\circ} \mathrm{C}$. The mainly reason is that during the radical copolymerization, when the final conversion is low, the monomer with a strong conjugacy is easier to polymerize than the others, styrene is such a hard monomer and the glass transition temperature of its homopolymer is $105^{\circ} \mathrm{C}$, so the $\mathrm{Tg}$ of copolymer will be a little higher than usual; on the contrary, when the final conversion is high, the system is able to polymerize according to the expectable proportion, the measured value and theoretical value of $\mathrm{Tg}$ match very well. However, the conversion of polymerization can not reach $100 \%$. In addition, Tg will increase because of the hydrogen bonds formed between the Components [7]. Some references [8-11] introduce that some additives and functional monomers will have a certain impact on glass transition temperature, therefore, there will be a difference between measured and expected value of $\mathrm{Tg}$ inevitably.

\subsection{The Latex Recipe of Different $\mathrm{Tg}$}

In this paper, the total mass of the monomer is fixed at $70 \mathrm{~g}$ and the ratio of two hard monomer (St and MMA) will not change at about 1:1, we changes the proportion of soft and hard monomer only. Table 4 shows the latex recipe with different $\mathrm{Tg}$ which are obtained at the condition of $\mathrm{A}_{2} \mathrm{~B}_{2} \mathrm{C}_{2}$.

Table 4 shows that when the final conversion is at a high level, the measured value and theoretical value of $\mathrm{Tg}$ matches very well, thus researcher can be able to forecast the $\mathrm{Tg}$ of polymer according to the proportion monomers.

\section{CONCLUSIONS}

1) The results of the orthogonal experimental shows that emulsifier $>$ initiator $>$ stirring speed in terms of their effects on conversion. And we get the best condition: emulsifier $4 \%$, initiator $0.6 \%$, stirring speed $180 \mathrm{rpm}$.

Table 4. Latex recipe with different $\mathrm{Tg}$.

\begin{tabular}{cccccc}
\hline & $\mathrm{BA}(\mathrm{g})$ & $\mathrm{MMA}(\mathrm{g})$ & $\mathrm{St}(\mathrm{g})$ & $\begin{array}{c}\text { theoretical } \\
\text { value of } \mathrm{Tg}\end{array}$ & $\begin{array}{c}\text { measured } \\
\text { value of } \mathrm{Tg}\end{array}$ \\
\hline $1 \#$ & 48 & 11 & 11 & $-20.0^{\circ} \mathrm{C}$ & $-18.3^{\circ} \mathrm{C}$ \\
$2 \#$ & 43 & 14 & 13 & $-10.0^{\circ} \mathrm{C}$ & $-9.3^{\circ} \mathrm{C}$ \\
$3 \#$ & 38 & 16 & 16 & $-3.0^{\circ} \mathrm{C}$ & $-2.2^{\circ} \mathrm{C}$ \\
$4 \#$ & 33 & 19 & 18 & $7.8^{\circ} \mathrm{C}$ & $9.8^{\circ} \mathrm{C}$ \\
$5 \#$ & 28 & 21 & 21 & $20.0^{\circ} \mathrm{C}$ & $20.4^{\circ} \mathrm{C}$ \\
$6 \#$ & 26 & 22 & 22 & $24.0^{\circ} \mathrm{C}$ & $25.2^{\circ} \mathrm{C}$ \\
$7 \#$ & 24 & 23 & 23 & $30.0^{\circ} \mathrm{C}$ & $32.7^{\circ} \mathrm{C}$ \\
$8 \#$ & 20 & 25 & 25 & $40.0^{\circ} \mathrm{C}$ & $42.7^{\circ} \mathrm{C}$ \\
$9 \#$ & 17 & 27 & 26 & $45.0^{\circ} \mathrm{C}$ & $46.9^{\circ} \mathrm{C}$ \\
\hline
\end{tabular}


2) There is a direct relationship between conversion and glass transition temperature, the improvement of the final conversion has made the measured value close to the theoretical value.

3) At the condition of high conversion, the measured value and theoretical value of $\mathrm{Tg}$ matches very well.

\section{REFERENCES}

[1] Yu, Y.B. and Zhang, Y.H. (1996) Studying progress on styrene-acrylate emulsion polymerization[J]. Chemical Industry and Engineering Progress, 2, 36-39.

[2] Wang, W.F., Li, S.X. and Liu, L.M. (2007) Preparation of low VOC waterborne epoxy-acrylate hybrid emulsion for wood coating [J]. Coating Industry, 37(11), 30-33.

[3] Pan, G.R., Wu, L.M. and Zhang, Z.Q. (2002) Synthesis and characterization of epoxy-acrylate composite latex [J]. Journal of Applied Polymer Science, 83(8), 1736-1743.

[4] Chen, C.F., Lee, K.H. and Chiu, W.Y. (2007) Synthesis and characterization of poly (butyl acrylate-methyl methacrylate)/polyaniline core-shell latexes [J]. Journal of Applied Polymer Science, 104(2), 823-830.
[5] Joensson, J.L., Hassander, H. and Jansson, L.H. (1991) Morphology of two-phase polystyrene/poly (methyl methacrylate) latex particles prepared under different polymerization conditions [J]. Macromolecules, 24(1), 126-131.

[6] Chen, R. (2007) Minimum temperature and polymer glass-transition temperatures [J]. Chemical Industry of Acrylic and Application, 20(1), 17-19.

[7] Gómez-Carracedo, A. and Alvarez-Lorenzo, C. (2003) Chemical structure and glass transition temperature of non-ionic cellulose ethers DSC, TMDSC [J]. Journal of Thermal Analysis and Calorimetry, 73(2), 587-596.

[8] Luo, H. and Wei, Z.G. (1997) Study on Tg of acrylic emulsion copolymer [J]. Fine Chemicals, 69, 42-45.

[9] Xu, J. and Chen, B. (2005) Prediction of glass transition temperatures of OLED materials using topological indices [J]. Journal of Molecular Modeling, 12(1), 24-33.

[10] An, J., Li, X.Y. and Zhu, X.W. (2007) Segment distributing of copolymer form microemulsion copolymerization of styrene and butyl acrylate [J]. Chinese Journal of Colloid \& Polymer, 25 (1), 1-2.

[11] Wang, C.C. and Bao, Q.Y. (2005) Acrylate coatings [M]. Chemical and Industry Publishing Company, Beijing. 